\title{
Intrinsic spin-orbit interactions in flat and curved graphene nanoribbons
}

\author{
M. P. López-Sancho, and M.C. Muñoz \\ Instituto de Ciencia de Materiales de Madrid, Consejo Superior \\ de Investigaciones Científicas, Cantoblanco, 28049 Madrid, Spain
}

(Dated: November 5, 2018)

\begin{abstract}
Recent theoretical and experimental works on carbon nanotubes and graphene samples have revealed that spin-orbit interactions, though customarily ignored in carbon-based materials, are more important and complex than it was thought. We study the intrinsic spin-orbit coupling effects on graphene nanoribbons, both flat and bent. Calculations are performed within the tight-binding model with the inclusion of a four-orbital basis set. Thereby the full symmetry of the honeycomb lattice and the hybridization of $\sigma$ and $\pi$ bands are considered. In addition to the zero-energy $\pi$ edge states, $\sigma$-derived edge states are found for the three investigated ribbon geometries. The $\sigma$ states are also spin-filtered and localized at the boundaries of the ribbons. The calculated spin-orbit splittings are larger for the $\sigma$ - than for the $\pi$-derived edge states. Due to this enhancement, spinorbit splittings of the $\sigma$-states reach values of the order of a few Kelvin. These spin-filtered edge states are robust under $\sigma-\pi$ hybridization and curvature effects.

PACS numbers: 73.20.-r, 73.22.-f, 73.23.-b
\end{abstract}

\section{INTRODUCTION}

The influence that geometry and size have on the electronic properties of carbon based materials has been already proved. Two dimensional graphene sheets, graphene stripes known as graphene nanoribbons (GNRs), or carbon nanotubes (CNTs) formed by rolling a graphene sheet unto a cylinder, due its unique electronic properties mainly to their geometries. Chirality and diameter determine the properties of CNTs, while the GNRs physics depends on its width and the shape of its edges ${ }^{1}$. This strong dependence of the electronic properties on the geometry arises from the peculiar structure of graphene with carbon atoms ordered in a honeycomb lattice, divided into two nonequivalent triangular sublattices, and confers these materials promising potential applications in electronics and spintronics. The low energy physics of carbon-based materials is governed by the states close to the Fermi energy in graphene. They correspond to the $\pi$-states near the nonequivalent $K$ and $K^{\prime}$ points at opposite corners of the hexagonal Brillouin zone (BZ), the Dirac points, at which the valence and conduction bands touch and present a conical energy spectrum.

Due to the low atomic number of carbon, spin-orbit interaction (SOI) was expected to be small. Nevertheless, several theoretical studies were devoted to investigate SOI effects in graphite ${ }^{2}$ and more recently in CNTs and graphene $\mathrm{e}^{3-8}$. In a k.p scheme and using a perturbation approach, it was shown that the SOI opens a gap in the energy bands crossing at the Fermi level and lifts the spin degeneracy for higher energy subbands ${ }^{3}$. In a previous work ${ }^{6}$, within a four-orbital tight-binding (TB) framework, we have demonstrated the chiral dependence of the intrinsic SOI effects, which can not be described by a continuum model. Spin-splitting is only possible for chiral CNTs due to the lack of inversion symmetry, while the spin-degeneracy remains for achiral CNTs. Furthermore, the recently measured asymmetric splittings of va- lence and conduction bands in a CNT quantum dot $\frac{9}{\underline{9}}$ are also explained within the same mode ${ }^{10-12}$ pointing out that curvature enhances the SOI strength. In fact, recent experimental measurements of the energy shifts caused by the SOI, have proved that the SOI effects in CNT quantum dots and graphene samples are not as small as predicted $^{9,13}$. Besides, since SOI is the key ingredient for the Quantum Spin Hall state, the new phase of matter proposed by Kane and Mele ${ }^{14}$ in graphene, the previously considered almost negligible SOI interaction in graphenebased nanostructures has risen to be a main topic in the condensed matter field ${ }^{15}-20$.

The electronic properties of GNRs have been intensively investigated due to the remarkable behavior of the zero-modes edge states ${ }^{21}-27$. Most of the works have been based either on the k.p or the effective $\pi$-orbital TB approaches, the simplest models that capture the physics of graphene, although some first-principles and $\mathrm{LCAO}^{28-30}$ calculations have also been reported. Furthermore, the effect of the SOI in graphene sheets and GNRs with different boundaries is currently attracting great interest and, in spite of the large amount of published works, many aspects are still hidden. Some numerical calculations based on multiorbital models 31 , and first principles ${ }^{32,33}$ can be found in the literature but, to our knowledge, no investigation of the SOI on flat and bent GNRs based on a $s p^{3}$ tight-binding model has so far been performed.

We address here the study of the intrinsic spin-orbit coupling effects on the electronic properties of GNRs with edges of different shapes: zigzag, Klein's bearded and armchair edges. We consider flat and bended ribbons to investigate the interplay between the SOI and the curved geometry and focus on the topological edge states, not only the well known $\pi$-derived states, but also those arising from $\sigma$-orbitals. We investigate how curvature influences the interplay of confinement and SOI in determining the topological character of the edge states. 
An explicit description of edge states requires a model that gives the energy bands through the entire BZ. Therefore, calculations are performed by exactly solving an empirical tight-binding Hamiltonian with a $s p^{3}$ basis set. The TB model handles equally all points in the BZ, which is not possible with the continuum model and is especially appropriated with regard to the treatment of the SOI. Besides, it takes into account the full symmetry of the honeycomb lattice, hence the threefold rotational symmetry and the trigonal warping are included. Nor external potentials or doping are taken into account, although the effect of the $\sigma$-derived edge states on the behavior of the spin Hall conductivity with the chemical potential would be discussed.

In addition to the $\pi$-edge states we found that $\sigma$ derived edge states are also present for all the boundary geometries of the GNRs studied. Further, as expected its energy is independent of GNR width and for a large interval of $\mathbf{k}$ values of the one dimensional BZ the $\sigma$ edge sates are located in a gap. Hence, they are likewise perfectly localized along the edges of the ribbon. Our main finding is that, when the SOI term is included in the Hamiltonian, independent of the GNR termination, the fourfold degeneracy of edge states, both of $\pi$ and $\sigma$ character, is lifted except at the time reversal invariant symmetry points $\Gamma$ and $K$ of the one dimensional BZ. They split into the spin filtered edge-states, which are robust upon $\sigma-\pi$ bands hybridization and, as expected for a topological state, are unaffected by curvature.

The paper is organized as follows: in Section II the details of the model are given. In Section III the results obtained for the different type of GNRs are described, and curvature effects are analysed by comparison of flat and curved geometries. Finally, we conclude in Section IV summarizing our results and drawing some conclusions.

\section{MODEL AND METHOD}

A ribbon consists of a stripe of graphene of infinite length and finite width. There are two prototypical shapes of the GNR edges, zigzag and armchair, they have a $\theta=30$ angle of difference in the cutting direction and the termination of a generic ribbon has a combination of the two types. We consider ribbons of three different kinds of terminations: zigzag, bearded and armchair, and define the width of the GNRs as $n$, where $n$ stands for the number of zigzag lines for the zigzag and bearded ribbons and for the number of dimer lines for the armchair ribbon 21 . The corresponding unit cell contains $N=2 n$ carbon atoms. Examples of the three types of ribbons are displayed in Figure 1. We do not consider here any reconstruction or relaxation of the edges 34,35 . Nevertheless, chemical and structural modifications of graphene edges are promising routes to design GNR based devices $20,25,27$.

Curvature effects are introduced by bending the ribbon along its width. The bending is realized without stretch-
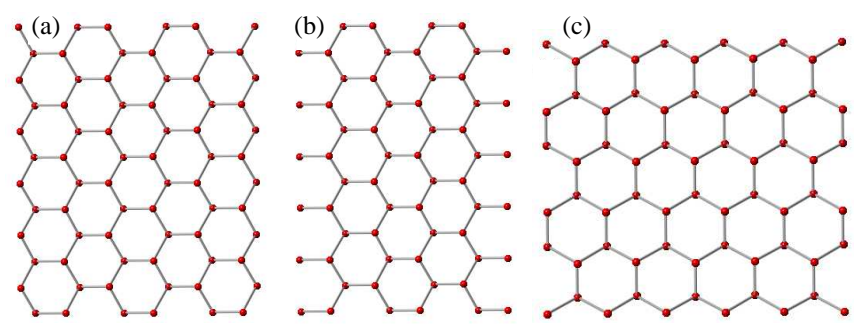

FIG. 1: Schematic lattice structure of graphene nanoribbons with: a)zigzag, b) bearded, and c) armchair edges.

ing, varying the atom coordinates to form an open cylinder. Different curvatures are obtained by changing the angles and diameters of the cylindrical configurations.

The electronic properties of the graphene nanoribbons are calculated from the one-electron Hamiltonian given by,

$$
H=H_{0}+H_{S O},
$$

$H_{0}$ is the spin-independent noninteracting Hamiltonian,

$$
H_{0}=\frac{\mathbf{p}^{\mathbf{2}}}{2 m}+V(\mathbf{r})
$$

and and $H_{S O}$ is the microscopic spin-orbit interaction term

$$
H_{S O}=\frac{\hbar}{4 m^{2} c^{2}} \mathbf{S} \cdot(\nabla V \times \mathbf{p})
$$

where $\mathrm{V}$ is the full crystal potential, $\mathbf{p}$ the electron momentum and $\mathbf{S}$ represents the spin operator.

We calculate the band structure of the graphene ribbons by the Slater-Koster ${ }^{36}$ empirical tight-binding (ETB) Hamiltonian. A four-orbital, $2 s, 2 p_{x}, 2 p_{y}$ and $2 p_{z}$, basis set is considered in order to include the conventional on-site approach for the intrinsic SO interaction. Within the TB approximation the $H_{0}$ term is written as,

$$
H=\sum_{i, \alpha, s} \epsilon_{\alpha}+\sum_{<i j>, \beta, s} t_{i j}^{\alpha, \beta} c_{i, s}^{\alpha+} c_{j, s}^{\beta}+H . c .
$$

where $\epsilon_{\alpha}$ represents the atomic energy of the orbital $\alpha,<$ $i j>$ stands for atomic sites of the honeycomb lattice and $c_{i, s}^{\alpha+}$ and $c_{i, s}^{\alpha}$ are the creation and annihilation operators of one electron at site $i$, orbital $\alpha$ and spin $s$, respectively.

Since the major contribution of the crystal potential, $\nabla V$, is near the atomic nuclei, the intrinsic SOI can be accurately approximated by a local atomic term of the form:

$$
H_{S O}=\frac{\hbar}{4 m^{2} c^{2}} \frac{1}{r_{i}} \frac{d V_{i}}{d r_{i}} \mathbf{L} \cdot \mathbf{S}=\lambda \mathbf{L} \cdot \mathbf{S}
$$




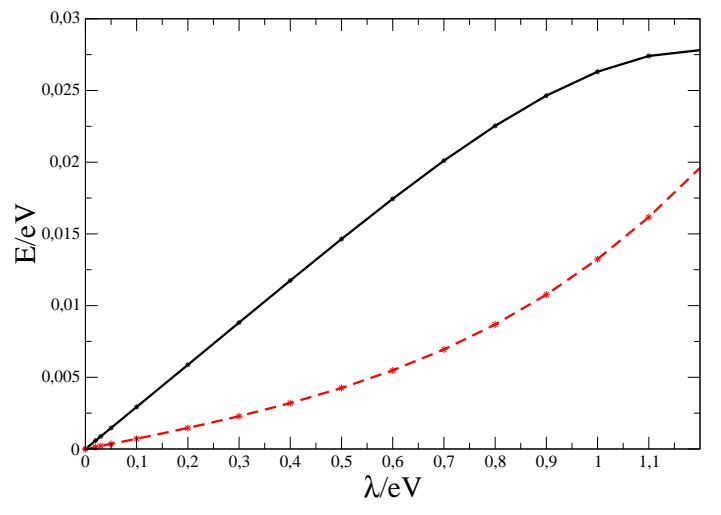

FIG. 2: (Color on line) Energy splitting for the conduction (circles) and valence (diamonds) bands of the $(61,0)$ zigzag $\mathrm{CNT}$ as a function of the value of the spin-orbit coupling constant $\lambda$. The solid(black), dashed(red) lines are a guide to the eyes.

where spherical symmetry of the atomic potential is assumed. $r_{i}$ is the radial coordinate with origin at the atom, $V_{i}$ the spherical symmetric potential about the same atom and $\mathbf{L}$ the orbital angular momentum. The atomic SO coupling constant $\lambda$ depends on the orbital angular momentum $\mathbf{L}$ and thus on the atomic orbital.

For the $s p^{3}$ model Hamiltonian, SO coupling occurs only among $p$ orbitals and neglecting nearest neighbor SO terms, the intrinsic SOI is described as an on-site interaction among the $p$-orbitals. In contrast, in the k.p and effective $\pi$-band models, the atomic SOI is treated by perturbation theory as a second-neighbor spin dependent hopping term ${ }^{3,7}$

$H_{S O}$ adds diagonal and off-diagonal spin-dependent matrix elements to the $8 N \times 8 N$ matrix Hamiltonian, where $\mathrm{N}$ is the number of carbon atoms of the unit cell and 8 comes from the four-orbital per spin TB basis set. Using the raising and lowering angular momentum operators, $L_{+}=L_{x}+i L_{y}$ and $L_{-}=L_{x}-i L_{y}$, respectively and the Pauli spin matrices, the complete Hamiltonian, $\mathrm{H}$, in the $2 \times 2$ block spinor structure is given by,

$$
H=\left(\begin{array}{cc}
H_{0}+\lambda L_{z} & \lambda L_{-} \\
\lambda L_{+} & H_{0}-\lambda L_{z}
\end{array}\right) .
$$

where the atomic-like spin-orbit term $H_{S O}$ has been added to SK-ETB spin-independent $H_{0}$ Hamiltonian. The diagonal terms act as an effective Zeeman field producing gaps of opposite signs at the $K$ and $K^{\prime}$ points of the BZ.

In the $s p^{3}$ TB model the non-vanishing matrix elements of $H_{S O}$ couple $2 p_{x}, 2 p_{y}$ and $2 p_{z}$ orbitals. These terms have been widely discussed previously, see for example Ref.37,38. $H_{S O}$ induces $\sigma-\pi$ hybridization and does not break the time reversal symmetry, therefore spin degeneracy can not be removed on systems with inversion symmetry.

The electronic properties of the GNRs are obtained by the exact diagonalization of the total Hamiltonian
H. The Tománek-Louie parametrization for graphite ${ }^{39}$, previously used to calculate SOI effects on CNTs, has been also employed in the present calculations. The TB parameters, taken from Ref 39 are : $\epsilon_{s}=-7.3 \mathrm{eV}$, $\epsilon_{p}=0.0 \mathrm{eV}, s s \sigma=-4.30 \mathrm{eV}, s p \sigma=4.98 \mathrm{eV}, p p \sigma=6.38 \mathrm{eV}$, $p p \pi=-2.66 \mathrm{eV}$, and specify the energy scale of the model.

The spin-orbit coupling strength of graphene is unknown and its exact value is under debate. Due to the small atomic number of carbon, it has been assumed to be very small and different values in the order of $\approx m e V$ have been given $7,8,14$. In order to have a quantitative estimate of the SOI induced splittings, we have evaluated $\lambda$ from the SO induced gaps experimentally measured in a CNT quantum $\operatorname{dot}^{9}$. In the experiment a zigzag small gap CNT of about $5 \mathrm{~nm}$ of diameter is probed using tunnelling spectroscopy. The anisotropic gaps measured at zero magnetic field are of $\approx 0.37 \mathrm{meV}$ for electrons and of $\approx 0.22 \mathrm{meV}$ for holes. The $(61,0) \mathrm{CNT}$ with a diameter of about $4.8 \mathrm{~nm}$ is a zigzag semiconducting tube of the $n=3 m+1$ family, so the SOI induced splitting is greater for the conduction than for the valence band ${ }^{10}$ as occurs in the experiment. Calculated SOI splittings for the conduction and valence bands are represented in Fig 2 for the $(61,0)$ nanotube as a function of $\lambda$. From the figure it is inferred that $\lambda \approx 3 \mathrm{meV}$ is needed in order to to obtain SOI splittings similar to those experimentally reported. This value is of the same order of magnitude of previous estimations $i^{7,8}$. In the figures we present results obtained with values of $\lambda$ in the range of $0.2-0.4 \mathrm{eV}$, for illustrative purposes. This range corresponds to $0.03-0.06$ in units of the $p p \sigma=6.38 \mathrm{eV}$ parameter.

\section{RESULTS}

The electronic properties of GNRs are derived from the band structure of graphene subject to a stripe geometry. The combination of the confinement due to the finite-size and the presence of boundaries yields the peculiar band structure of GNRs. The truncation of inter-atomic bonds caused by the borders gives rise to the appearance of edge states, which are strongly dependent on the atomic termination of the GNR. Moreover, the energy subbands associated to the intrinsic band structure of the graphene sheet are also dependent on the boundary conditions of the GNR. Calculations have been carried out in GNRs of different geometries and widths. In order to avoid the coupling between edge states localized at each boundary, ribbons of more than 50 chains are considered.

\section{A. Zigzag graphene nanoribbons}

In zigzag graphene nanoribbons (ZGNRs) the atoms of each edge belong to the same sublattice and opposite edge atoms are of different sublattices. In the absence of SOI, the band structure of ZGNRs presents together with the subbands originating from the two- 


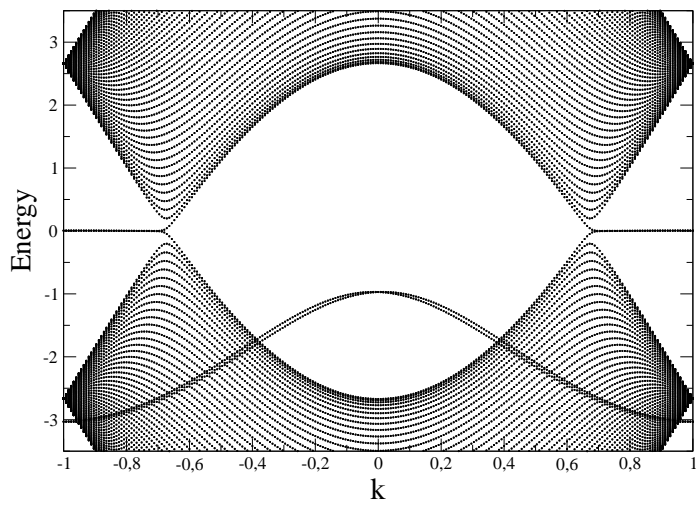

FIG. 3: Band structure of the zigzag ribbon $n=60$ obtained with SOI $\lambda=0.4$. $k$ are in units of $\pi$

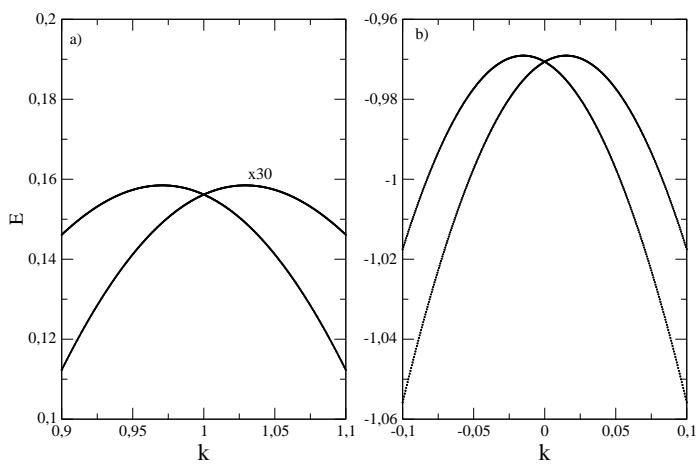

FIG. 4: Zoom of the band structure of the ribbon $\mathrm{N}=60$ with $\lambda=0.4$ for the two edge states, left around $k=1$ for the zero edge states (energies are multiplied by 30 ) and right in the region of $k=0$ for the $\sigma$ derived band. $k$ are in units of $\pi$

dimensional graphene electronic structure, zero-energy $\pi$-orbital derived states at the $\frac{2}{3} \pi<k<\pi$ interval. These states, with wave functions localized at the edges, form flat bands and give rise to a peak in the density of states at the Fermi energy being crucial for magnetic instabilities 21,40 . Besides, between 1 and $3 \mathrm{eV}$ below the Fermi energy, $\sigma$-orbital derived edge band appears in the $s p^{3}$ TB calculation. These states, which are missed in one- $\pi$ band models, disperse along the BZ and their wave function amplitude is also fully localized at the edge atoms around $\mathbf{k}=\mathbf{0}$ and $\mathbf{k}= \pm \pi$, where the ZGNRs have energy gaps. It is worth mentioning that ribbons of different widths have been studied, from $n=10$, to $n=200$ and edge states, both $\pi$ and $\sigma$, appear at similar energies independent of the ribbon width.

To analyze the result of the SOI in ZGNRs, the band structure of the $n=60$ flat ZGNR with $\lambda=0.4 \mathrm{eV}$ is shown in Figure 3 in the range of $-\pi \leq \mathbf{k} \leq \pi$. The spectrum remains gapless and the degeneracy of edge sates, originated from both $\pi$ - and $\sigma$ - orbitals, is partially lifted. A zoom of the dispersion relation of the $\pi$ and $\sigma$ edge states around the time-reversal invariant $\mathbf{k}= \pm \pi$ and $\Gamma$ points, respectively, are presented in Figure 4. The SOI shifts down in energy the edge states giving a small
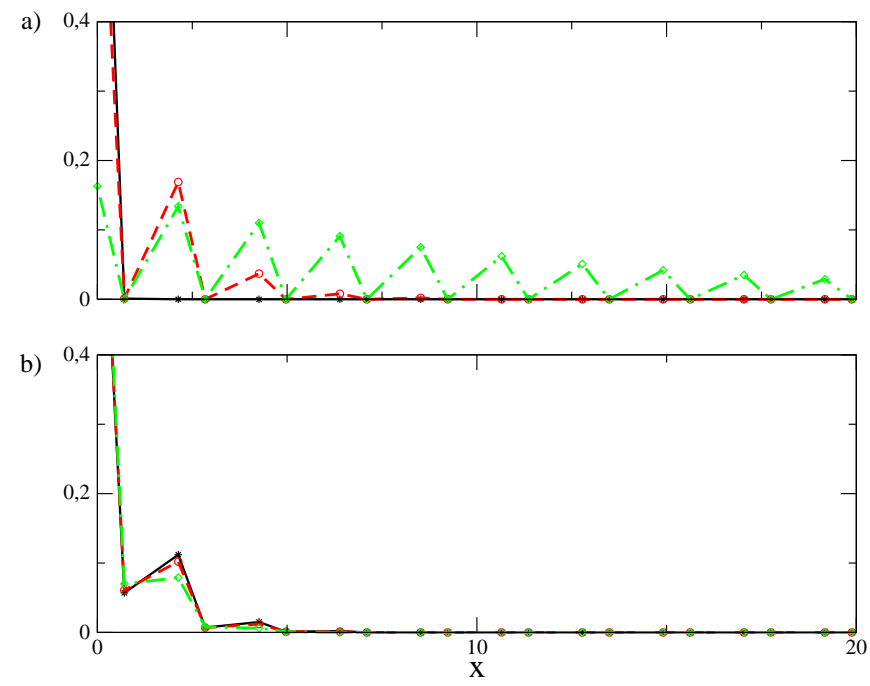

FIG. 5: (Color on line)Wave function amplitude of the localization versus atomic coordinates accross the ribbon width for the two edge states states represented in Fig. 4. a) for the state at the Fermi level at $k=\pi$ (black stars), $k=0.85 \pi$ (red circles) and $k=0.7 \pi$ (green diamonds) b) for the $\sigma$ derived state at $k=0$ (black stars), $k=0.15 \pi$ (red circles) and $k=0.3 i \pi$ (green diamonds). Black solid, red dashed and green dott-dashed lines are guides for the eyes.

dispersion to the $\pi$ flat bands. It also lifts partially the fourfold degeneracy of both $\pi$ and $\sigma$ edge states, which become twofold degenerate, except at the $\mathbf{k}= \pm \pi$ and $\mathbf{k}=0 /(\mathbf{k}= \pm \pi)$ points of the BZ, respectively. Each edge state splits into two degenerate Kramers doublets, with linear dispersion in a very small $k$-region around the crossing and forward and backward mover states with opposite spin. Since degenerate states are confined at different edges of the ZGNR, the resulting states with opposite spin are localized in either edge. Thus two independent spin-filtered edge states are at each boundary of the ribbon. Hence, for a given energy the ZGNR has four conducting channels spatially separated, an extreme of the ribbon contains a forward mover with a given spin $\mathbf{S}$ and a backward mover with opposite spin, $-\mathbf{S}$, and conversely for the other extreme of the ribbon. Note, that the energy splitting of the $\pi$ and $\sigma$ edge states differs almost by a factor of 30 .

The localized character of these states can be observed in Figure 5, where the wave function amplitudes are represented against the atomic coordinates across the width of the $n=60$ ribbon, for three different values of $\mathbf{k}$ in the BZ. The wave function amplitude of both $\pi$ and $\sigma$ states is fully localized in the edge atoms in a large $\mathbf{k}$ interval around the $\mathbf{k}= \pm \pi$ and $\Gamma$ points, respectively. The localization length of the zero-energy state increases with $k$ and eventually, for $\mathbf{k}$ close to $\pm \frac{2 \pi}{3}$, where it merges with the bulk bands, becomes extended. Further, the spatial localization correlates with the $\pi-\sigma$ hybridization induced by the SOI. At $\mathbf{k}= \pm \pi$, where the state is composed of purely $\pi$ orbitals, its wave function is fully localized in the 
edge atoms, moving towards the $\Gamma$ point, i.e. $\mathbf{k}= \pm 0.9 \pi$, the localization starts to decrease toghether with an admixture with $\sigma$ orbitals. Therefore, the presence of the SOI and consequently of the $\pi-\sigma$ hybridization, slightly reduces the localization length of $\pi$ edge states. Analogously, the $\sigma$ edge states show an almost pure $\sigma$ orbital character and a strong localization at the borders of the ribbon for both $\Gamma$ and $\mathbf{k}= \pm \pi$ points. The presence of a small $\pi$ contribution, for $\mathbf{k}$ values different from the high symmetry points, gives rise to the increase of the localization length.

Due to spin-orbit coupling $\mathbf{S}$ is not longer a good quantum number and the eigenfunctions are a linear combination of spin-up and spin-down states. As stated above, each extreme of the ribbon contains two states, forward and backward movers, with opposite spin $\mathbf{S}$. The calculated expectation value of $\langle\mathbf{S}\rangle$ shows that the spin orientation of both $\pi$ and $\sigma$ edge states is almost perpendicular to the graphene plane at the time-reversal invariant $\mathbf{k}=\pi$ and $\Gamma$ points. The deviation from the perpendicular axis is smaller than $1 \%$. The orientation axis, although depends on the magnitude of $\mathbf{k}$, slightly change in the $\mathbf{k}$ interval in which edge states remain spatially localized. When $\sigma-\pi$ hybridization becomes relevant and states deslocalize, the in-plane component has a finite value and the spin orientation axis of extended states forms an angle with the graphene plane. Furthermore, the expectation value of the orbital angular momentum $<\mathbf{L}>$ for edge states is almost zero, indicating the quenching of the orbital angular momentum.

Finally, because of the estimated small value of $\lambda \approx 3$ $m e V$, the corresponding SOI splitting of $\pi$-states is only of the order of $0.17 \times 10^{-3} \mathrm{meV}$, which gives temperatures in the range of $10^{-3} \mathrm{~K}$, in agreement with previous estimations. Nevertheless, for the new $\sigma$-derived edge states the calculated splitting is much larger, $0.51 \mathrm{meV}$, which results in temperatures of the order $\approx 5 \mathrm{~K}$ that, although small, is experimentally attainable and thus, the topological protected spin-filtered states of graphene could be observed.

\section{B. Klein bearded graphene nanoribbons}

In this termination, as in the case of zigzag edges, all atoms of one end belong to one sublattice, and those of the opposite end, to the other sublattice. The Klein's edge could be formed by bonding an additional $\mathrm{C}$ atom to every edge site of the zigzag ended ribbon ${ }^{41}$. Zigzag and Klein ribbons differ in the number of cut bonds.

Figure 6 shows the dispersion relation of a $n=60$ ribbon with this geometry without and with the SOI effect. The Klein boundary produces as well edge states localized at the ends of the ribbon, with the same behavior of those of the zigzag nature, although the zeroenergy flat bands lie at the central region of the $\mathrm{BZ}^{21,23}$, $-\frac{2 \pi}{3} \leq \mathbf{k} \leq \frac{2 \pi}{3}$. Besides the flat zero edge $\pi$ states, there are two new edge states of $\sigma$ character. In the absence of
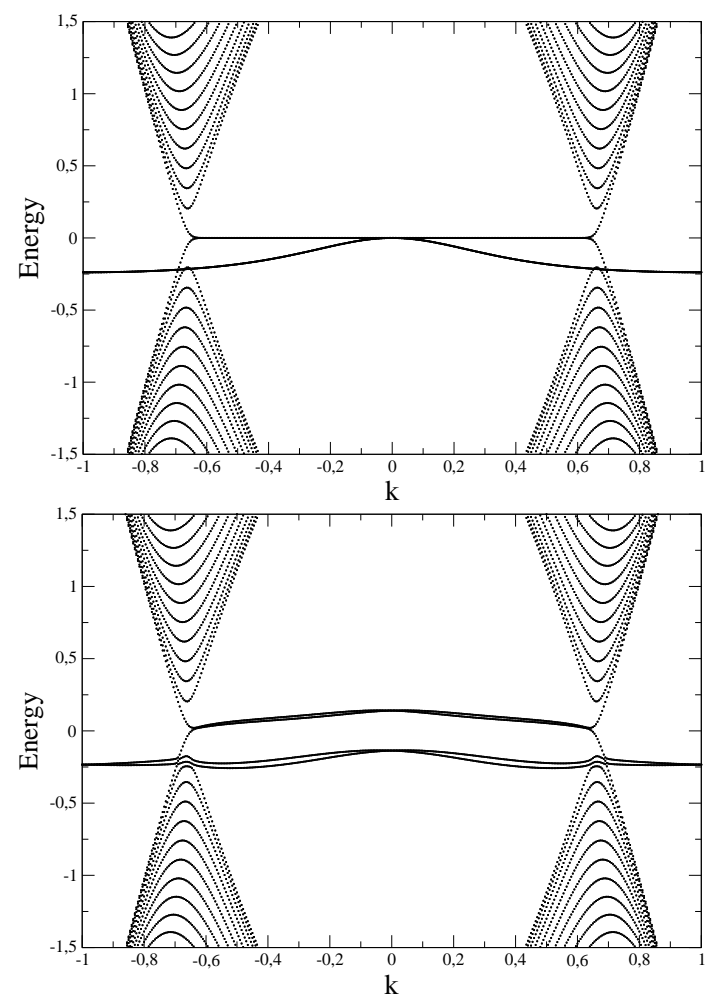

FIG. 6: Band structure of the bearded edge $\mathrm{N}=60$ ribbon without(with) SOI left(right). $k$ are in units of $\pi$

SOI, one of the $\sigma$ states, not shown in the figure, lies well below the Fermi energy, at around $-4 \mathrm{eV}$, mixing with the bulk bands, and showing a weak dispersion along the whole BZ. The other $\sigma$ state, lies at zero energy at the $\Gamma$ point, but presents energy dispersion, lowering its energy as $|\mathbf{k}|$ increases, lying at $-0.23 \mathrm{eV}$ for $\mathbf{k}= \pm \pi$. The flat bands are of pure $\pi$ character, and are localized in the outermost atoms at either edge of the ribbon. Also the wave function amplitude of the the dispersive band of mainly $\sigma$ nature is strongly localized at the edge atoms, $\approx 0.91 \%$ at the extreme atoms. The spin-orbit coupling, besides to induce the splitting into two Kramer's doublets of the edge bands, has drastic effects on the energy and dispersion of both $\pi$ and $\sigma$ bands. While $\pi$ states acquire a small dispersion, a large energy shift and flattening of the $\sigma$ bands occurs. These effects originate from the $\pi-\sigma$ hybridization induced by the SOI and depend on the value of the coupling strength. For example, the zero energy state was at $\mathbf{k}=0.2 \pi$ pure $\pi$-like in the absence of SOI term, but has around $1.1 \%$ of $\sigma$ components when the SOI term is considered with the $\lambda=3 \mathrm{meV}$ coupling. As shown in Figure 6 bottom, the SOI shifts the edge bands, inducing a separation at $\Gamma$, for $\lambda=0.4 \mathrm{eV}$, of 276 $m e V$ which varies almost linearly with the value of $\lambda$ and therefore it reduces to $7.38 \mathrm{meV}$ estimated when $\lambda=3$ meV.

Moreover, the SOI induced energy splitting of edge states is of $\approx 0.38 \mathrm{meV}$ and $0.18 \times 10^{-3} \mathrm{meV}$ for the lower and higher energy bands respectively at $k= \pm 0.2 \pi$. The 
difference of the splittings is due to the different orbital compositions of the bands. The energy splitting of the $\pi$ and $\sigma$ edge states is of the same order than in the zigzag ribbons. The dependence of the orbital composition on $\mathbf{k}$ explains the different splitting magnitudes at different regions of the BZ. The extended bulk subbands states, associated to the intrinsic band structure of the graphene sheet are, in the presence of spin-orbit coupling, still doubly degenerate due to the combination of time reversal and inversion symmetries.

The average $\langle\mathbf{S}>$ and $<\mathbf{L}>$ values, analogous to those of zigzag ribbons, point out the counterpropagation nature of the edge states and give a spin quantization axes almost perpendicular to the sheet axes.

\section{Armchair graphene nanoribbons}

Armchair edges are formed by homogeneous lines of dimers where atoms belonging to the two different sublattices alternate. The electronic properties of these ribbons present a strong dependence on their width, as it happens for zigzag CNTs. The armchair ended ribbons are metallic when its width $W=(3 M-1) d$, where $M$ is a positive integer and $d$ is the $\mathrm{C}-\mathrm{C}$ atom distance. For other values of $W$ the armchair ribbons (AGNRs) are semiconductors with direct band gaps at $\Gamma$ which are inversely proportional to its width $\frac{28,42}{2}$. They follow a family behavior as it is found for zigzag CNTs 10,30 , where metallic behavior is obtained in a sequence of period 3. However, zigzag CNTs are metallic for $n=3 M$ due to the different periodic boundary conditions imposed by the cylindrical geometry. Ribbons, with both metallic and semiconductor behavior, of different widths are calculated in order to clarify how the SOI affects both electronic structures.

Metallic AGNRs

Armchair ribbons of width $W=(2 M-1) d$ present a band structure with two states crossing at the Fermi energy at the $\Gamma$ point of the BZ. Figure 7 shows the band structure of the $n=62$ ribbon calculated with SOI and $\lambda=0.4$. In the absence of spin-orbit coupling, the crossing states are only spin degenerate, have pure $\pi$-orbital character, show linear dispersion and their wave functions are extended throughout the whole ribbon width. The inclusion of the SOI term in the Hamiltonian opens a small gap at $\Gamma$, for the ribbon $n=62$ is of $\approx 10^{-4}$ $m e V$ for $\lambda=3 \mathrm{meV}$, which increases as the width of the ribbon decreases 31 . Further, it changes the orbital composition of the linear states allowing for a small proportion about $5 \%$, of $\sigma$ orbitals and the pure spin nature is not conserved. Nevertheless, the two linear dispersing states remain twofold degenerate and keep its extended character. These states have been found to persist in the presence of intrinsic spin-orbit interactions as spin filtered states localized on the ribbon edges within the low-energy Dirac model $\stackrel{15}{ }$, In the present model, in order to obtain localization of these states, we need unphysical high values of $\lambda$ of the order of $2.8 \mathrm{eV}$.
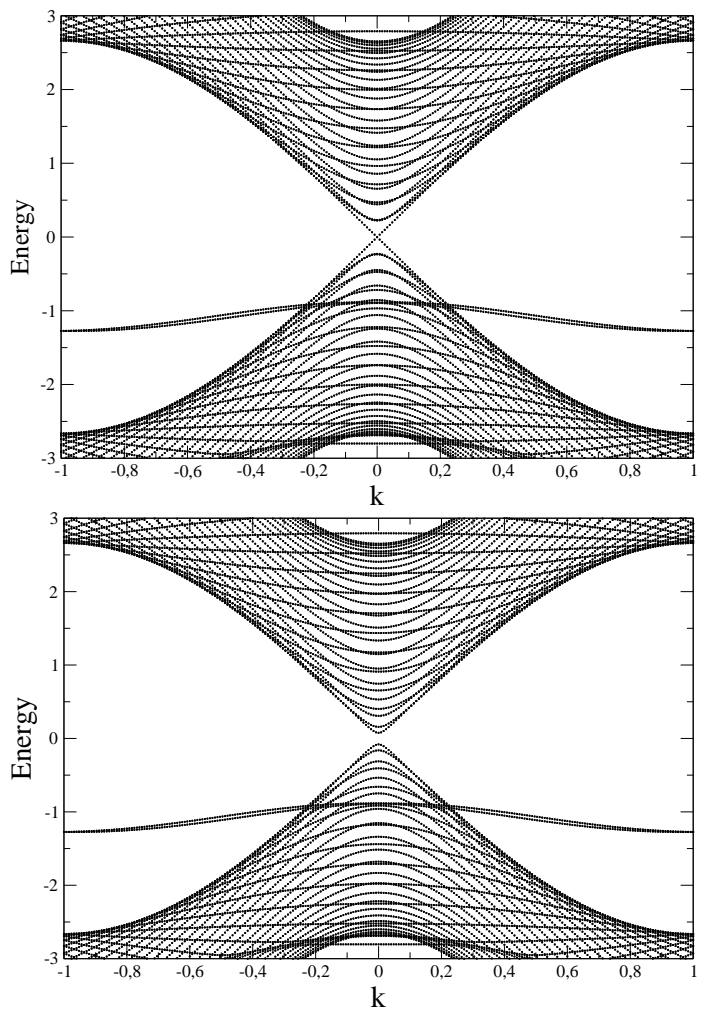

FIG. 7: Band structure of the armchair $\mathrm{N}=62$ metallic ribbon (top) and $\mathrm{N}=60$ semiconductor ribbon (bottom) with SOI $\lambda=0.4 . k$ are in units of $\pi$

Edge states composed mainly of $\sigma$ orbitals and fourfold degeneracy appear below the Fermi level. They lie at $-1.27 \mathrm{eV}$ at the $k= \pm \pi$ points of the $\mathrm{BZ}$, are dispersive and fully localized at the edge atoms of the ribbon. At $k \approx \pm 0.3 \pi$ merge with the bulk bands, but keep its fourfold degeneracy and localized character. The wave function amplitude is confined to the outermost atoms, although, at the zone center, localization occurs at both ends of the ribbon. These $\sigma$-orbital derived states are truly surface states appearing at the same energy independently of the ribbon width, as we have confirmed calculating AGNRs of up to $n=122$ and $n=242$.

The $\sigma$ derived edge states, upon SOI inclusion, split into two doublets, except at the time-reversal invariant $k= \pm \pi$ points, where they remain fourfold degenerate. The value of the splitting varies with $k$ reaching a maximun value of $\approx 0.36 \mathrm{meV}$ at $k= \pm 0.5 \pi$ for $\lambda=3 \mathrm{meV}$. These states keep the localization at the boundary atoms, although their orbital composition presents now a small proportion of $\pi$ orbitals. The so-called bulk subbands of the ribbon mantain always their double degeneracy.

\section{Semiconductor armchair GNRs.}

The bottom of Fig. 7 shows the band structure corresponding to the semiconductor ribbon $\mathrm{N}=60$, with SOI $\lambda=0.4$. The energy gap at $\Gamma$ is of $0.156 \mathrm{eV}$ almost equal to the value obtained without SOI. The $\sigma$-orbital derived edge states and bulk related subbands present the same 
features as in the metallic ribbons. Even the SOI energy splitting of the Krammers doublets are of the same order than in the previous case.

\section{Curvature effects}

Next we analize the effect of curvature. As stated above, in the curved geometry bond stretching is not allowed along the ribbon. Even the distances between the carbon atoms at the edges are not changed in any of the different terminations studied. The lattice structure of the ribbon is isotropically bended along the width, $x$-direction, while the bonding distances, and therefore the hoppings, between atoms in the $y$-direction are not modified.

Curvature induces hybridization between $\pi$ and $\sigma$ orbitals. In fact several works have shown that curvature enhances the SOI effects in CNTs $6,10,11,31$. The curvature induced $\sigma-\pi$ hybridization is related with the amplification of the SOI effects for small diameter tubes. However, curvature effects turn out to be weaker for ribbons than for $\mathrm{CNTs}^{31,43}$. The cylindrical shape of the tubes impose periodic boundary conditions that confer symmetries to the wave functions which do not hold in the open geometries. As for the flat geometry, a critical value of the width -slightly greater- is needed in order to avoid the coupling of edge states in the bent ribbons. Above the critical value of the width of the ribbon, the behavior of the edge states found in the flat ribbons is not affected by the curvature. The localized nature of the edge states remains upon bending, only in some cases the localization lenght increases. In order to illustrate the differences between flat and curved geometries the electronic structure of the $n=10$ zigzag ribbon, both flat and bent, are depicted in Figure 8

In the absence of SOI, flat and bent ZGNRs only differ by a small variation of the subband dispersions. In both geometries edge states, $\pi$ - and $\sigma$-orbital derived, appear at similar energies although the orbital composition of the $\pi$ states is slightly changed with a small admission of $\sigma$ orbitals in the curved ribbon. The zero-energy dispersionless states, in the bent geometry, present a very weak dispersion. Both, the $\sigma$-orbital admission and the dispersion, increase as $\mathbf{k}$ increases, and merge with the bulkbands at a $\mathbf{k}$ smaller than in the flat geometry. These differences are clear in Figure 8 where crossing of the edge state occurs in the flat $n=10 \mathrm{ZGNR}$ band structure while, due to the hybridization, anticrossing is observable in the curved case. SOI effects are similar in both geometries, although a slight increase of the splittings appears in the curved ribbon.

In order to compare with the $n=10$ ribbons, Figure 9 represents the band structure of the armchair $(5,5)$ carbon nanotube, both without and with SOI. The $(5,5)$ $\mathrm{CNT}$, could be thought as formed by joining 44 the two borders of the curved ZGNR $n=10$. Due to the breaking of the rotational symmetry, the number of bands is greater in the ribbon than in the tube. While the point group of the ZRGN, both flat and bended, is the $C_{3}$ group of graphene, the $(5,5)$ tube belongs to the $D_{5 d}$ group. The corresponding irreducible representations are ten $1 \mathrm{D}$ for the $C_{3}$ and two $1 \mathrm{D}$ and four 2D for the $D_{5} d^{2}$. Consequently, considering spin all the subbands are twofold degenerated in the ZGNR and two are twofold and four fourfold degenerated in the CNT, out of the ten bands appearing in the energy interval shown in Figures 8 and 9 (see for example Ref. ${ }^{6}$ ). However, in both ribbons and NT, the lower bands crossing at the Fermi level are only spin degenerate.

The different degeneracy accounts for the different consequences that curvature has in the ribbons and in the CNTs in the presence of SOI. As it is well known, SOI removes all degeneracies compatible with time reversal symmetry and parity. Since ZGNR states are only spindegenerated, large splittings comparable to those of the fourfold degenerate bands of the NT are not observable. Note that the band structure shown in Figure 8 corresponds to the major possible bended ZGNR, which forms an open cylinder with a curvature identical to that of the CNT. In fact, curvature effects are also small in ZGNR in the presence of the SOI. They reduce to a slight increase of the energy splitting, see for example the second subband. Therefore, enhancement of the SOI strength, analogous to that shown for CNT, does not occur in ribbons.

The effects of curvature on the bearded ribbons are very similar, inducing changes in the orbital compositions of the edge states as well as a very weak dispersion in the flat bands. At $\Gamma$ the $\sigma$-orbital derived edge zero-energy states are shifted down in energy by curvature effects, but the shift in energy decreases with the ribbon width. Also, there is an small increase of the energy splittings in the curved geometry when the SOI is included.

On the other hand, the main effect of curvature on the armchair ribbons is to induce a small gap at $\Gamma$ on the metallic ribbons between the two linear states crossing at the Fermi energy. As well, in both metallic and semiconducting AGNRs, the SOI induced energy splittings of the $\sigma$ derived edge states are slightly greater on the curved than in the flat geometry.

Curvature also results in a variation of the spin orienation axis and in an increase of the orbital angular momentum. The expectation value of $\langle\mathbf{S}\rangle$ for the edge states is no longer perpendicular to the graphene sheet, but always an in-plane contributation turns the spin axis to form an angle with the ribbon plane. Further, $\langle\mathbf{L}>$ increases by more than two orders of magnitude with respect to that of the corresponding flat ribbon. Nevertheless, for all the ribbons studied it is still much smaller than the orbital moment attributed to CNTs due to their cylindrical geometry.

In general, the major effect of curvature is on the edge states, in the absence of SOI and for flat ribbons with widths greater than 50 chains, the wave functions of the degenerate edge states are fully localized at either end of 

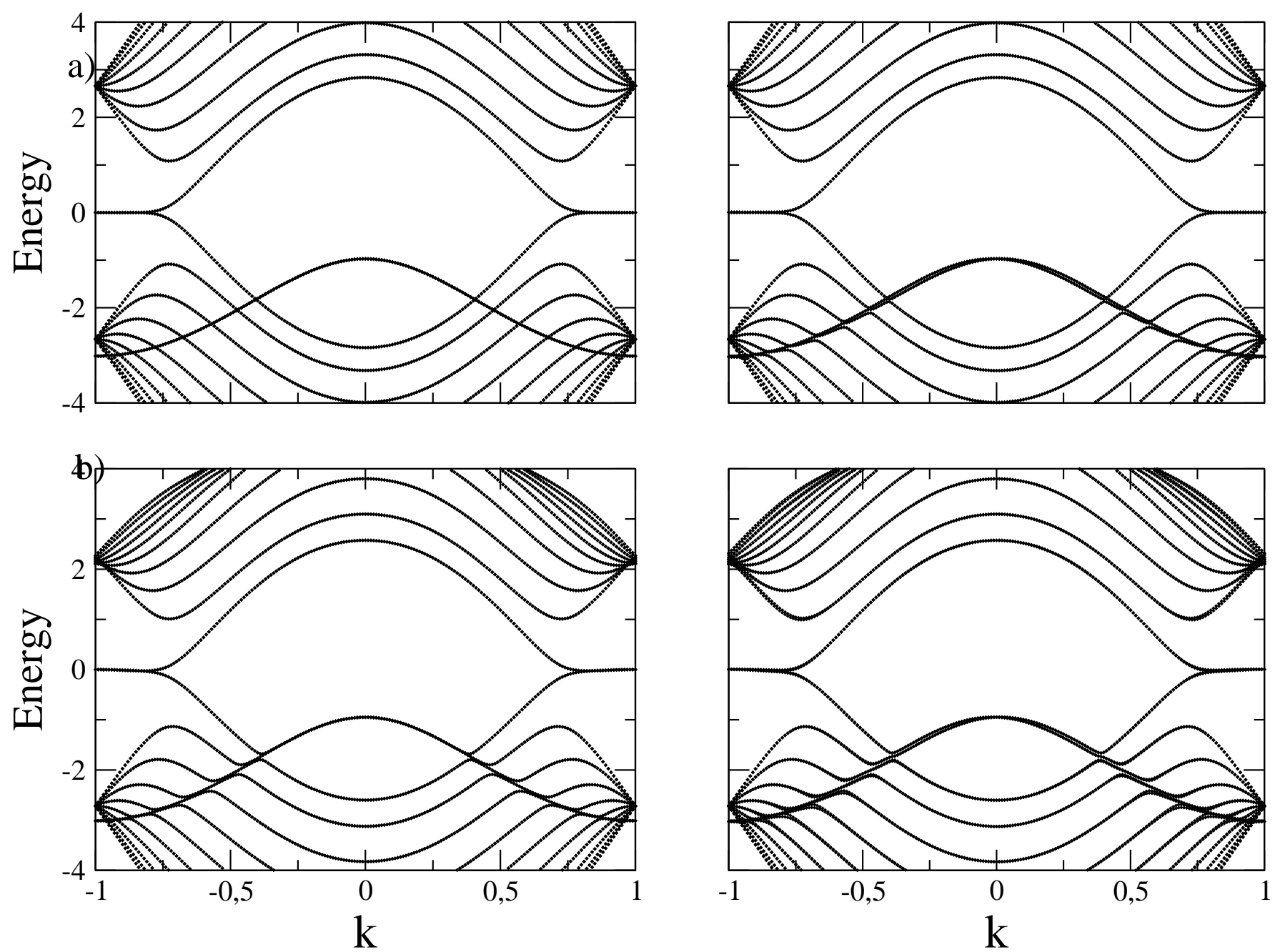

FIG. 8: Left:Band structure of the $\mathrm{N}=10$ zigzag nanoribbon a) flat, b) curved, calculated with $\lambda=0$ (left) and $\lambda=0.4 \mathrm{eV}$ (right).

the ribbon, thus in the presence of SOI they become spinfiltered states. However, for curved ribbons the states localized at the left and right ends interact and accumulates at both edges, preventing the formation spin conducting chanels.

\section{DISCUSSION AND CONCLUSIONS}

We have studied the spin-orbit interaction effects on the electronic structure of graphene nanoribbons taking into account the actual discreteness of the lattice. The energy subbands associated to the graphene intrinsic electronic bands remain doubly degenerate even when the SOI term is included in the Hamiltonian. The atomic lattice structure satisfies spatial inversion symmetry and, in the presence of time reversal symmetry, the intrinsic spinorbit coupling does not break the spin degeneracy ${ }^{6,14}$.

The result of the spin-orbit coupling is much more pro- nounced in the edge states and particularly in those with a large contribution of $\sigma$ orbitals, as it happens in CNTs and bilayer graphene ${ }^{10,45}$. In fact, besides recovering previous results on the edge states originated from $\pi$ orbitals, a better understanding of the interplay between the intrinsic spin-orbit coupling and the lattice geometry is reached. It is found that for the flat zero bands that appear at $\frac{2 \pi}{3} \leq \mathbf{k} \leq \pi$ and $-\frac{2 \pi}{3} \leq \mathbf{k} \leq \frac{\mathbf{2} \pi}{\mathbf{3}}$ in the zigzag and bearded ribbons respectively, the SOI induced splitting is greater in the regions of the BZ where the larger hybridization with $\sigma$ orbitals takes place.

The inclusion of $\sigma$ orbitals in the basis set gives rise to edge states missed in one-band calculations. Edge states originated from the $\sigma$-orbitals, have been found in the three types of ribbons investigated, lying below the Fermi energy. These states are localized in the extreme atoms at different regions of the BZ (around $\mathbf{k}=\mathbf{0}$ and $= \pm \pi$ ). As it occurs for the $\pi$-derived states, SOI lifts its fourfold degeneracy except at the $\mathbf{k}= \pm \pi$ and $\Gamma$ points protected 

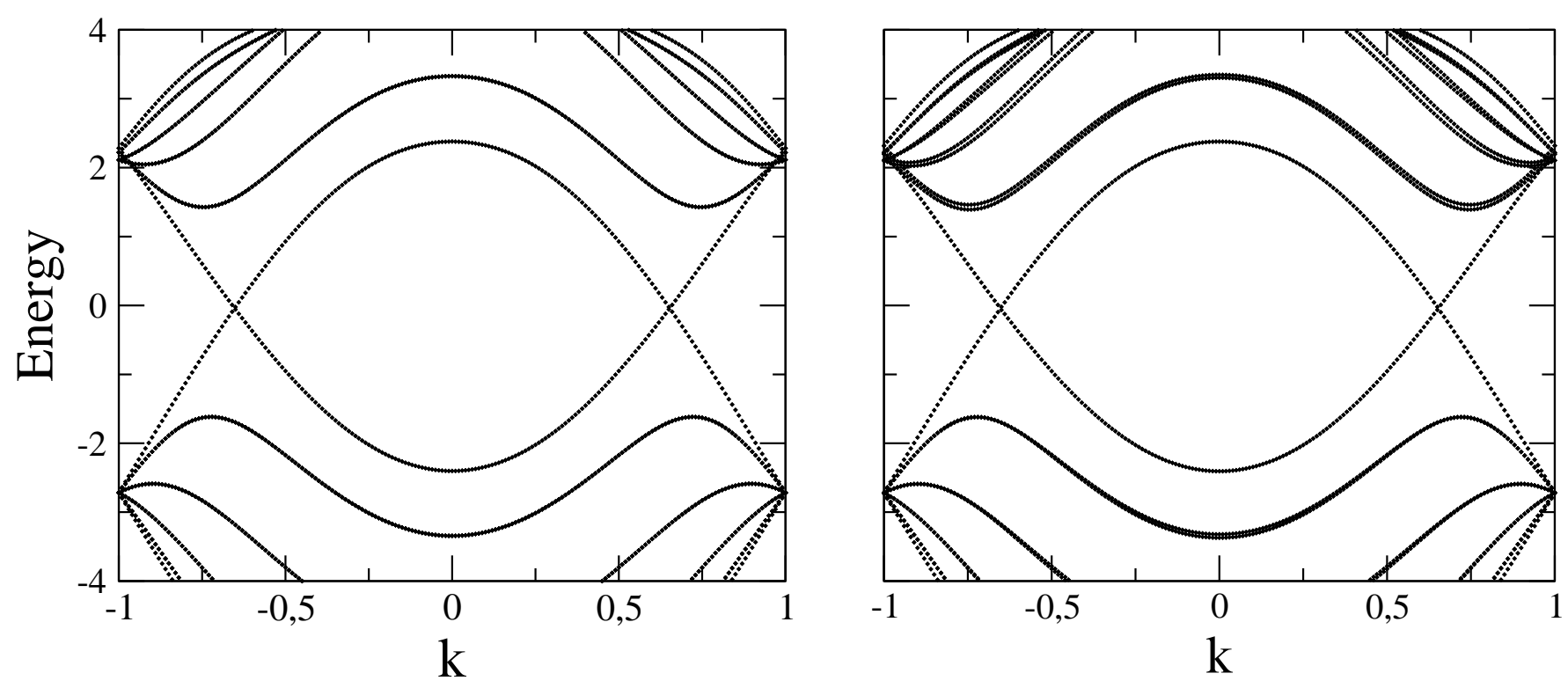

FIG. 9: Band structure of the armchair $(5,5)$ carbon nanotube calculated with $\lambda=0$ (left) and $\lambda=0.4 e V$ (right).

by time reversal symmetry. A relevant result is that the energy splitting is greater, more than and two orders of magnitude, for the $\sigma$ states than for the $\pi$ derived states. This result confirms those found for $\mathrm{CNTs}_{\mathrm{s}} \frac{10}{}$ and for a graphene bilayer where, due to the mixing between $\pi$ and $\sigma$ bands by interlayer hoppings, the spin-orbit coupling is about on order of magnitude larger than in a single layer $\stackrel{45}{ }$.

While the energy spin-orbit coupling induced splittings are of the order of $10^{-3} \mathrm{meV}$, equivalent to $\mathrm{T} \approx 0.001 \mathrm{~K}$ for the $\pi$ derived edge bands, the edge states originated from $\sigma$ orbitals present splittings of the order of $10^{-1}$ $m e V \quad i . e . \mathrm{T} \approx 4-5 \mathrm{~K}$. These results suggest that $\sigma$ states would be particularly appropiated to observe the spin currents associated with the spin-filtered edge states.

In the present work, Fermi energy is taken at zero energy and neutral graphene is considered, but appropriate chemical doping or external gates may change this value to tune the energy of the $\sigma$ edge states. In general, they lie around $1 \mathrm{eV}$ below the Fermi level, although for the Klein GNRs the $\sigma$ derived states are only a few meV away from $E_{F}$. Therefore, they are easily accesible by applying an external potential, which confers Klein nanoribons the abbility to exhibit the quantum spin Hall effect.

Since curvature is known to strongly enhance the spinorbit induced effects on carbon nanotubes, we have considered both flat and curved nanoribbons and showed the different behaviour that open and close boundary conditions impose.

The effects produced by the SOI term and by curvature would in principle be similar since both induce $\sigma-\pi$ hybridization and lift degeneracies. Nevertheless, curvature, for the bearded and armchair ribbons, lifts some of the degeneracies at the $\Gamma$ point, while SOI does not. This result can be understood considering the mapping of the two-dimensional graphene bands on the axial direction of the ribbons: while for the zigzag ribbons the Dirac points are projected at $\mathbf{k}= \pm \frac{\mathbf{2} \pi}{\mathbf{3}}$ for armchair $K$ and $K^{\prime}$ points are mapped to $\Gamma$. The curvature of the lattice affects the bands around the $\Gamma$ region of the $\mathrm{BZ}$ in the armchair ribbons, in contrast, for the zigzag termination, the main effects of curvature occur in the vicinity of $k= \pm \frac{2 \pi}{3}$. Analogously in CNTs curvature induces a gap in the primary metal $n=3 q$ zigzag tubes while armchair CNTs are metallic.

The interplay between curvature and SOI effects in nanoribbons are not as significant as in $\mathrm{CNTs}^{31}$ The boundary conditions imposed by the cylindrical shape of CNTs confer rotational symmetry which, alongside to the bipartite nature of the honeycomb lattice, is in the origin of the unusual electronic properties of CNTs. Orbital magnetic moments 10-20 times larger than the Bohr magneton have been observed in suspended CNTs 46 , attributed to semiclassical electron orbits encircling the tube circumference, whose diameter is much larger than the radii of the atomic orbitals $\underline{46}$. This behavior does not occur in the bent ribbons, where no rotational symmetry holds. The spin-orbit coupling effect in bent ribbons is mostly due to the increased $\pi-\sigma$ hybridization. Thus, both curvature and SOI contribute to the increase of the orbital angular momentum and to deviate the spin orientation from the perpendicular direction to the graphene sheet. Finally, it is found that curvature does not break the chiral symmetry of the edge states and its effects on the localization reduce to a small increase of the localization length.

In summary, we estimate the magnitude of the intrinsic spin-orbit coupling to be of $\approx 3 \mathrm{meV}$ and although small, we have demostrated that, under certain geometries, and experimental conditions the Quantum Spin Hall state 
predicted in graphene should be experimentally observed in nanoribbons at temperatures of the order of 3-4 K. Further, the spin-filtered edge-states are robust and unaffected by curvature. Ours results highlight the importance of symmetry to understand the spin-orbit coupling effects in graphene nanoribbons.

\section{ACKNOWLEDGMENTS}

The authors thank L. Brey, L. Chico and F. Guinea for discussions. This work has been partially supported by the Spanish DGES under grants MAT2006-05122, FIS2008-00124 and MAT2009-14578-C03-03.
1 A. H. Castro Neto, F. Guinea, N. M. R. Peres, K. S. Novoselov, and A. K. Geim, Rev. Mod. Phys. 81, 109 (2009).

2 G. Dresselhaus and M. S. Dresselhaus, Phys. Rev. 140, A401 (1965).

3 T. Ando, J. Phys. Soc. Jpn. 69, 1757 (2000).

${ }^{4}$ M. Entin and L. Magarill, Phys. Rev. B 64, 085330 (2001).

5 A. D. Martino, R. Eggert, K. Hallberg, and C. Balseiro, Phys. Rev. Lett. 88, 206402 (2002), ; J. Phys.:Condens. Matter 16, S1437 (2004).

${ }^{6}$ L. Chico, M. López-Sancho, and M. Muñoz, Phys. Rev. Lett. 93, 176402 (2004).

7 D. Huertas-Hernando, F. Guinea, and A. Brataas, Phys. Rev. B 74, 155426 (2006).

8 H. Min, J. E. Hill, N. A. Sinitsyn, B. R. Sahu, L. Kleinman, and A. H. MacDonald, Phys. Rev. B 74, 165310 (2006).

${ }^{9}$ F. Kuemmeth, S. Ilani, D. Ralph, and P. McEuen, Nature (London) 452, 448 (2008).

10 L. Chico, M. P. López-Sancho, and M. C. Muñoz, Phys. Rev. B 79, 235423 (2009).

11 J.-S. Jeong and H.-W. Lee, Phys. Rev. B 80, 075409 (2009).

12 W. Izumida, K. Sato, and R. Saito, J. Phys. Soc. Jpn. 78, 074707 (2009).

13 I. Gierz, J. Dil, F. Meier, B. Slomski, J. Osterwalder, J. Henk, R. Winkler, C. Ast, and K. Kern (2010), arXiv/1004.1573.

14 C. L. Kane and E. J. Mele, Phys. Rev. Lett. 95, 226801 (2005).

15 M. Zarea and N. Sandler, Phys. Rev. Lett. 99, 256804 (2007).

16 M. Zarea, C. Büsser, and N. Sandler, Phys. Rev. Lett. 101, 196804 (2008).

17 M. Zarea and N. Sandler, Phys. Rev. B 79, 165442 (2009).

18 Z. Wang, N. Hao, and P. Zhang, Phys. Rev. B 80, 115420 (2009).

19 R. van Gelderen and C. M. Smith, Phys. Rev. B 81, 125435 (2010).

20 M. J. Schmidt and D. Loss, Phys. Rev. B 81, 165439 (2010).

${ }^{21}$ M. Fujita, K. Wakabayashi, K. Nakada, and K. Kusakabe, J. Phys. Soc. Jpn. 65, 1920 (1996).

22 S. Ryu and Y. Hatsugai, Phys. Rev. Lett. 89, 077002 (2002).
23 N. M. R. Peres, A. H. Castro Neto, and F. Guinea, Phys. Rev. B 73, 195411 (2006).

24 L. Brey and H. A. Fertig, Phys. Rev. B 73, 235411 (2006).

${ }^{25}$ H. Raza and E. C. Kan, Phys. Rev. B 77, 245434 (2008).

${ }^{26}$ K. Wakabayashi, Y. Takane, M. Yamamoto, and M. Sigrist, Carbon 47, 124 (2009).

27 K. Wakabayashi, S. Okada, R. Tomita, S. Fujimoto, and Y. Natsume, J. Phys. Soc. Jpn. 79, 034706 (2010).

28 Y.-W. Son, M. L. Cohen, and S. G. Louie, Phys. Rev. Lett. 97, 216803 (2006).

29 J. C. Boettger and S. B. Trickey, Phys. Rev. B 75, 121402 (2007).

30 G. Zhang, X.W.and Yang, J. Phys. Chem. C 113, 4662 (2009).

31 S. Onari, Y. Ishikawa, H. Kontani, and J.-i. Inoue, Phys. Rev. B 78, 121403 (2008).

32 L. Pisani, J. A. Chan, B. Montanari, and N. M. Harrison, Phys. Rev. B 75, 064418 (2007).

33 M. Gmitra, S. Konschuh, C. Ertler, C. Ambrosch-Draxl, and J. Fabian, Phys. Rev. B 80, 235431 (2009).

34 X. Jia, M. Hofmann, V. Meunier, B. Sumpter, J. CamposDelgado, H. Romo-Herrera, J.M.and Son, A. Hsieh, YP. Reina, J. Kong, M. Terrones, and M. Dresselhaus, Science 323, 1701 (2009).

35 C. Girit, J. Meyer, R. Erni, M. Rossell, C. Kisielowski, L. Yang, C.-H. Park, M. Crommie, M. Cohen, S. Louie, et al., Science 323, 1705 (2009).

36 J. C. Slater and J. F. Koster, Phys. Rev. 94, 1498 (1954).

37 D. Chadi, Phys. Rev. B 16, 790 (1977).

38 S. Gallego and M. Muñoz, Surface Sci. 423, 324 (1999).

39 D. Tománek and S. G. Louie, Phys. Rev. B 37, 8327 (1988).

40 M. A. H. Vozmediano, M. P. López-Sancho, T. Stauber, and F. Guinea, Phys. Rev. B 72, 155121 (2005).

41 D. Klein, Chem. Phys. Lett. 217, 261 (1994).

42 M. Ezawa, Phys. Rev. B 73, 045432 (2006).

${ }^{43}$ F. de Juan, A. Cortijo, and M. A. H. Vozmediano, Phys. Rev. B 76, 165409 (2007).

44 H. Santos, L. Chico, and L. Brey, Phys. Rev. Lett. 103, 086801 (2009).

45 F. Guinea, New J. Phys. 12, 083063 (2010).

46 E. Minot, Y. Yaish, V. Sazonova, and P. McEuen, Nature (London) 428, 536 (2004). 